\title{
Variations in Intensity Statistics for Representational and Abstract Art, and for Art from the Eastern and Western Hemispheres
}

\author{
Daniel J. Graham and David J. Field
}

Department of Psychology

Cornell University

Ithaca, NY 14853 USA

\begin{abstract}
Two recent studies suggest that natural scenes and paintings show similar statistical properties. But does the content or region of origin of an artwork affect its statistical properties? We addressed this question by having judges place paintings from a large, diverse collection of paintings into one of three subject matter categories using a forced-choice paradigm. Basic statistics for images whose categorization was agreed by all judges showed no significant differences between those judged to be "landscape" and "portrait/still-life," but these two classes differed from paintings judged to be "abstract." All categories showed basic spatial statistical regularities similar to those typical of natural scenes. A test of the full painting collection (140 images) with respect to the works' place of origin (i.e., provenance) showed significant differences between Eastern works and Western ones, differences which we find are likely related to the materials and the choice of background color. Although artists deviate slightly from reproducing natural statistics in abstract art (compared to representational art), the great majority of human art likely shares basic statistical limitations. We argue that statistical regularities in art are rooted in the need to make art visible to the eye, not in the inherent aesthetic value of natural scene statistics, and we suggest that variability in spatial statistics may be generally imposed by manufacture.
\end{abstract}

Keywords: Natural Scenes, Vision, Art, Representation, Abstract Art, Statistics, Scale Invariance, Paintings, Painting Statistics, Image Search, Art Provenance

\section{INTRODUCTION}

Images of the natural world have been found to share statistical regularities, including spatial frequency amplitude spectrum shape and slope (Field, 1987; Burton and Moorhead, 1987), sparseness (Field, 1994) and local contrast (e.g., Frazor and Geisler, 2006). That natural images are predictable in a statistical sense suggests the visual world to which the human visual system has adapted over evolution occupies a limited subspace within the space of all possible images. Arguments based on efficiency considerations have shown that visual system design (particularly early vision) can take advantage of this redundancy (Laughlin, 1981; Srinivasan et al., 1982; Atick and Redlich, 1992; see Graham and Field, 2007a for a review) and that simple models of 
visual systems that exploit these redundancies can predict observed cell response properties (e.g., Olshausen and Field, 1996, Bell and Sejnowski, 1997). But few in this field have considered how such statistical regularities are manifest in human-created visual presentations, and in particular, in art.

Earlier work (Graham and Field, 2007b) has suggested that a large and diverse sample of roughly luminance-calibrated paintings shows strong statistical regularities that are quite similar to statistical regularities of natural scenes. This study found that 124 digitally scanned paintings from the Herbert F. Johnson Museum of Art at Cornell University, representing a roughly equal share of works from the Western and Eastern hemisphere, showed the same spatial correlation structure (i.e., Fourier spatial frequency amplitude spectrum) as that seen in natural scenes, though the fall-off of this relationship was significantly different for the paintings and the natural scenes. Redies et al. (2007) obtained similar results: this study found that spectra of a large and diverse sample of graphic art are more similar to spectra of natural scenes than they are to spectra of scientific illustrations, or to spectra of close-up views of common objects. The amplitude spectrum measures the relative contribution of different Fourier components to the image as a whole. It is related by a Fourier transform to the autocorrelation function, which measures correlation in neighboring pixels' intensities. ${ }^{1}$ Colloquially, the amplitude spectrum describes the proportion of large-scale structure and fine detail in an image. The fall-off in amplitude plotted on $\log -\log$ axes goes as $1 / f^{k}$, where $f$ is spatial frequency and $k$ is about 1.2 for natural scenes (Tolhurst et al., 1992; Field, 1993) and about 1.4 for paintings (Graham and Field, 2007b). The paintings, in other words, have a slightly steeper slope, i.e., they have relatively more low-frequency (large scale) amplitude compared to natural scenes. A related study by Taylor et al. (1999) showed that for Jackson Pollock's drip paintings of the 1950s, the paint layer's outlines display fractal-like scale invariance and that Pollock's work showed characteristic fractal dimension statistics at different points in his career. But whereas the tests by Taylor et al. (1999) relate to the fractal shape of paint mark outlines obtained after thresholding,

\footnotetext{
${ }^{1}$ Many researchers express the Fourier decomposition as a power spectrum. The amplitude spectrum is the square root of the power spectrum, and the slope of the amplitude spectrum is simply half the slope of the power spectrum.
} 
the results of Graham and Field (2007b) and Redies et al. (2007) relate to fractal-like multi-scale structure in the variation of tones over space. In particular, the latter studies suggested that nearly all paintings show $1 / f$ scaling of contrasts across spatial frequency in terms of their surface intensity maps. This is true regardless of the pigment application technique used in the works. That is, fractal-like scaling is a property not unique to paint boundary edges in Pollock's paintings - it is rather a characteristic of the great majority of paintings and, indeed, of natural scenes.

Fractal or scale-invariant contrast statistics in images may require a slope of 1.0 though there is active discussion regarding how such $1 / f$ structure can arise. Field (1994) argued that a selfsimilar array of 2D functions which increase exponentially in number with decreasing scale can produce $1 / f$ structure and Ruderman (1997) argued that a similar distribution of functions that allow occlusions can also produce such structure. Balboa et al. (2001) have argued that exponential scaling of the number of objects that comprise a scene is not necessary for producing a $1 / f$ amplitude spectrum. Indeed, Field and Brady (1997) noted that a fractal edge with the right scaling (i.e., an edge with a length that increases in proportion to decreasing scale) can also produce $1 / f$ structure in the $2 \mathrm{D}$ amplitude spectrum. A simple straight edge is insufficient (resulting in a rotationally averaged 2D amplitude spectrum of $1 / f^{2}$ ). However, it should be emphasized that objects with fractal edges - e.g., Mandelbrot or Julia sets — are not required to produce scale-invariant contrast statistics. Nor does 1/f scaling in 2-D amplitude spectra guarantee fractal edges or complete self-similarity.

Given that the visual system is well matched to the statistics typical of the natural world, it is perhaps no surprise that painters should reproduce those statistics in their work. Since painters create their art for human viewing it makes sense that they should on average conform to these regularities. Redies (2007) and others have argued that the similarity between natural scene statistics and art statistics is shaped by a universal aesthetic preference for natural statistics. While this is an interesting possibility that we are currently studying, here we argue for greater consideration of the importance of perceptibility and manufacture in accounting for this similarity (see Discussion). 
It should be noted that it is not possible to reproduce many of the luminance properties of natural scenes on canvas. As Graham and Field (2007b) noted, paintings are limited in the ratio of the most luminant point to the least luminant point (i.e., dynamic range), compared to natural scenes: paintings have a dynamic range of roughly 30:1 while scenes often exceed 1000:1. This difference is related to the number of illuminants for natural scenes (many) and for art (typically one). Graham and Field (2007b) argued that artists use global nonlinear luminance compression - in a manner analogous to that employed by photoreceptors - as a way to compensate for these dynamic range limitations.

Here we test statistical properties related to provenance. We also use a simple forced-choice classification scheme for the paintings in order to compare typical statistics across content classes and to test whether abstract subject matter is more or less likely to resemble natural scenes in terms of statistics, compared to other classes of paintings. In particular, for the first study, we separate the images by provenance to investigate cultural influences on art statistics. For the second study, we are interested in how a rough approximation of the range of depths in representational images can affect basic statistics, and how abstract content affects these statistics. The statistical measures are used to identify regularities in grey-scale pixel intensity distributions and in spatial structure. This study and future work with higher order statistics could offer new methods of image search and discrimination (see, e.g., Motoyoshi et al., 2007, for an approach to discriminating surface properties using intensity statistics; see also Torralba and Oliva, 2003, for studies of natural image classification using low-level statistics).

\section{STUDY 1: Painting Provenance}

\section{Methods}

The images used are from the same source as Graham and Field (2007b), the Herbert F. Johnson Museum of Art, Cornell University, though the size of the database has been expanded. Details about these images can be found in Graham and Field (2007b). Briefly, the 140 paintings used were all scanned by the same museum photographer using the same equipment and procedure. They are a fairly random sample representing about $10 \%$ of the museum's diverse collection of paintings. Paintings of Eastern provenance (Asia) represent $51 \%$ of the works, 
Western works (Europe and the Americas) form 49\% of the total, and together the works span approximately 900 years of art history. Provenance classification is based on metadata provided for each image by the museum (see Fig. 1). We calculated the first four statistical moments of the pixel intensity histograms (mean, variance, skewness, kurtosis) as well as the modal intensity for all images using intensity distributions for the whole image. We also calculated amplitude spectrum slope based on the central $818 \times 818$ pixel patch of each image (in order to compare across images, we used an $818 \times 818$ patch, which is the largest square that can be extracted from all images in our collection). Tests showed that choosing a randomly selected square patch produced the same statistical regularities as did choosing the central patch. These calculations relate to basic luminance distribution statistics and Fourier components for the art; there are hosts of other statistics one could measure that may be relevant, e.g., wavelet statistics.

[Fig. 1]

Results

Mean statistics for the two groups are shown in Table 1. While the mean intensity was significantly lower for Western works $(\mathrm{p}<0.0001)$, this group had a roughly 1.9 times higher variance than the Eastern works $(\mathrm{p}<0.0001)$. Both the mean and variance are a function of the scaling applied to the images so we normalized both groups' intensities to their respective means. In this case, the variance was 3.2 times higher $(p<0.0001)$ for the Western works (all other statistics are unchanged under normalization). This is equivalent to saying that the ratio of the standard deviation to the mean for the two classes of paintings is 1.8 times greater for the Western works compared to Eastern works (see Eqn. 1).

$$
1.8 \times \frac{S D(\text { East })}{\text { Mean }(\text { East })} \approx \frac{S D(\text { West })}{\text { Mean }(\text { West })}
$$

Though the sparseness (kurtosis) of the pixel intensities was not significantly different for images of Eastern and Western provenance, there were significant differences in skewness and 
amplitude spectrum slope for the two groups, with $\mathrm{p}$ values of $\mathrm{p}<0.0001$ and $\mathrm{p}<0.002$, respectively.

\begin{tabular}{|l|l|l|l|l|l|l|l|}
\hline Classification & $\begin{array}{l}\text { Number } \\
\text { of } \\
\text { Images }\end{array}$ & $\begin{array}{l}\text { Mean } \\
\text { (standard } \\
\text { error) }\end{array}$ & $\begin{array}{l}\text { Mode } \\
\text { (standard } \\
\text { error) }\end{array}$ & $\begin{array}{l}\text { Variance } \\
\text { (standard } \\
\text { error) }\end{array}$ & $\begin{array}{l}\text { Skewness } \\
\text { (standard } \\
\text { error) }\end{array}$ & $\begin{array}{l}\text { Pixel } \\
\text { Sparseness } \\
\text { (standard } \\
\text { error) }\end{array}$ & $\begin{array}{l}\text { Amplitude } \\
\text { Spectrum } \\
\text { Slope } \\
\text { (standard } \\
\text { error) }\end{array}$ \\
\hline $\begin{array}{l}\text { Eastern } \\
\text { Hemisphere }\end{array}$ & 72 & $\begin{array}{l}134.8^{*} \\
(3.13)\end{array}$ & $\begin{array}{l}137.5^{*} \\
(5.67)\end{array}$ & $\begin{array}{l}1721.6^{*} \\
(99.8)\end{array}$ & $\begin{array}{l}-0.314^{*} \\
(0.119)\end{array}$ & $\begin{array}{l}1.15 \\
(0.373)\end{array}$ & $-1.19^{*}$ \\
\hline $\begin{array}{l}\text { Western } \\
\text { Hemisphere }\end{array}$ & 68 & $\begin{array}{l}103.5 \\
(5.91)\end{array}$ & $\begin{array}{l}90.53 \\
(10.70)\end{array}$ & $\begin{array}{l}3251.1 \\
(260)\end{array}$ & $\begin{array}{l}0.428 \\
(0.137)\end{array}$ & $\begin{array}{l}0.950 \\
(0.347)\end{array}$ & -1.27 \\
\hline
\end{tabular}

Table 1. Pixel intensity statistics for art of the Eastern and Western hemispheres, with standard error shown in parentheses. Asterisks denote significant differences $(p<0.05)$ between mean statistics for Eastern and Western works.

Despite the fact that the skewness was different for the two groups, the sparseness of the pixel intensities was not significantly different. Skewness describes how far to the left or right of the mean of a distribution the majority of the samples lie; negative skew is found when more samples are to the left of the mean, positive skew is found when more samples are to the right of the mean. In general, natural scenes' high sparseness is largely due to high positive skew in their roughly log-normal intensity distributions. Paintings have a far smaller dynamic range and skewness than natural scenes (see Graham and Field, 2007). Here we hypothesize that the difference in skew between images of the Eastern and Western hemisphere is related to the background color of the images, since the background appears to account for the majority of the images' pixels. In particular, we observed that the Western works generally had a light foreground and dark background while the Eastern works generally had a dark foreground and light background. Such differences should be reflected in the mode of the intensities. As expected, we found that the modal intensity (from among the 256 available in 8-bit greyscale images) for the Eastern works was significantly higher than that for the Western works, with $\mathrm{p}<$ 0.0002 .

Discussion 
Results from our sample indicate that paintings from the Eastern and Western hemispheres differ significantly in terms of the normalized variance and the skewness of their pixel intensity distributions, and in terms of their amplitude spectrum slope. This difference in normalized variance suggests Western painters may tend to maximize the variance of their intensities for a given mean, which could be related to the strategies Western artists use to represent a range of strongly varying illuminations. Differences in skewness are attributed to the typical choice of background color for images in our database from the two hemispheres, as shown by the significant difference in the mean modal intensity for the two groups.

Amplitude spectra for Eastern works in our sample were significantly shallower than those of Western works. This may be a result of the media typically used in the production of the work. Eastern painters in our sample - especially the large proportion of $19^{\text {th }}$ century Indian paintings - tend to employ water-based pigments and fine brushes, whereas the Western works tend to employ oil paints, larger brushes and techniques like feathering. It is also possible that these differences are rooted in cultural notions of pictorial representation. A more detailed study of techniques and cultural influences could potentially disentangle these effects.

\section{STUDY 2: Painting Content}

\section{Methods}

Six judges (1F) were asked to classify the 140 images (presented in greyscale) as either "landscape," "portrait/still-life," or "abstract" using a forced-choice paradigm. The landscape and portrait/still-life discrimination was intended to reveal statistical regularities related to the represented range of depths. The abstract category was meant to identify statistical regularities related to non-representational content. Though there are limitless ways to categorize art by content, and though not all paintings would necessarily fit comfortably in these three categories, our method allows an approximate discrimination among paintings with a large implied range of depths, a small implied range of depths, and no implied range of depths.

Statistical analysis was carried out for images whose classification was agreed by all judges. The first four statistical moments were calculated for intensity distributions across the whole 
greyscale image; spatial frequency amplitude spectrum slope was again calculated for the $818 \mathrm{x}$ 818 pixel square at the center of each image.

[Fig. 2]

Results

Judges agreed on the classifications of 57 of the 140 images (see Fig. 2). There were no significant differences in distributions of any of the first four statistical moments (mean, variance, skewness, kurtosis) among the classes, nor between concrete works (landscape plus portrait/still-life) and abstract ones. For the amplitude spectrum slope, a significant difference was found between abstract and concrete categories $(\mathrm{p}<0.03)$, though both the landscape and portrait/still-life categories separately approached significant differences with the abstract works $(\mathrm{p}=0.058$ and $\mathrm{p}=0.054$, respectively). Mean values are given in Table 1 (see Fig. 3).

\begin{tabular}{|l|l|l|}
\hline Classification & Number of Images & Amplitude Spectrum Slope (standard error) \\
\hline Landscape & 19 & $-1.26(0.0387)$ \\
\hline Portrait/Still-Life & 26 & $-1.25(0.0300)$ \\
\hline Abstract & 12 & $-1.13(0.0614) *$ \\
\hline
\end{tabular}

Table 2. Table shows how amplitude spectrum slope varies with image content as determined by a forced-choice test. Standard error is shown in parentheses.

[Fig. 3]

\section{Discussion}

In our diverse though biased sample, we found only one significant difference between these content-based classes (concrete vs. abstract). This suggests that regardless of subject matter-and even when the subject matter does not correspond to real-world objects or scenesartists generally reproduce the regular statistics of the visual world in their paintings. Though art that is not strictly representational (i.e., works characterized as abstract here) can be produced 
with some reliance on random processes (e.g., such artistic movements as automatism, Dada and abstract expressionism), works in this class are hardly random in a statistical sense, at least for our sample. That is, despite their disconnection from concrete imagery and despite the possibility of elements of randomness in their production, abstract works show similar statistical regularities as representational works. Moreover, basic spatial statistics for art are very similar to those of natural scenes (see Graham and Field, 2007b), though the portraits, landscapes and abstract works each had significantly different mean slopes compared to the mean slope of the natural scene collection (1.40) in Graham and Field (2007b), with $\mathrm{p}<0.0002, \mathrm{p}<0.003$, and $\mathrm{p}<0.0001$, respectively.

Such regularity suggests that in order to produce a work fit for display in a museum, artists on average recreate the statistical regularities of the natural world. As noted in Graham and Field (2007b), this does not mean that all paintings must conform to these regularities in order to be deemed art. It merely suggests that the ubiquity of the statistical regularities in the natural world is generally sufficient to cause an artist to be unaware of their presence in paintings. In particular, the amplitude spectrum slope of the natural world — which is related to the proportion of largescale structure and fine detail - is simulated even by artists who do not attempt to portray a scene from the natural world, as in abstract works.

It is possible that abstract art shows spatial statistics similar to natural scenes in order to suggest the presence of real-world objects, even if no specific objects are depicted. As noted in Rogowitz and Voss' (1990) “cloud watching” experiment, when observers view computergenerated fractal "cloud" images, whose edges display fractal dimensions like those typical of natural images, the observers spontaneously report seeing namable objects. This suggests that in abstract art, as with fractal "cloud watching," spatial statistics that resembles those of natural scenes could promote the perception of object-like structure in non-representational images.

It is not clear why the amplitude spectrum slopes of non-representational works in our study were significantly shallower than those of representational works. This difference may relate to shading, which is used to denote shadows along edges in representational works, but is generally absent from non-representational works. Shading would tend to make edges softer and thereby lead to the steeper amplitude spectrum we observe for representational works. Abstract works as 
a group may also be concerned with an array of similarly sized forms, whereas representational works may tend to show one larger form (i.e., the painting's subject) surrounded by many smaller forms, which could also contribute to the difference in amplitude spectrum slope.

\section{GENERAL DISCUSSION}

The major findings of this paper are the following: for a large and diverse sample of paintings from a major university museum collection, there are systematic variations in the proportion of large- and small-scale detail (mean amplitude spectrum slope) for images with different psychophysically-assigned content identifiers, and for images produced in the two hemispheres. When classified by provenance, paintings also showed significant variations in mean pixel intensity skewness, which we find is likely related to the background tone typically used in the two hemispheres.

Our findings of a systematic difference in the modal tone, and the related difference in skewness by provenance in our sample stand in contrast to our finding of no significant differences in skewness related to image content. Of course, this could be a result of the smaller sample size used in the image content experiments. But the fact that the relatively small sample used in the image content studies could reveal differences in spectral content suggests that our sample was large enough to reveal first-order statistical differences among the image types.

Generalizing beyond our sample would require more data but based on our statistical analysis we suggest that Western artists of the past millennium typically choose to paint dark backgrounds and light foregrounds while Eastern painters typically choose light backgrounds and dark foregrounds. ${ }^{2}$ Artists of the Western hemisphere may also have introduced differences in their spatial statistics relative to Eastern artists by dint of their preferred medium (i.e., oilbased pigments). ${ }^{3}$

\footnotetext{
${ }^{2}$ There are of course exceptions to such trends: Japanese lacquer works typically have deep black backgrounds and lighter-toned foregrounds.

${ }^{3}$ Interestingly, Voss and colleagues have found that examples of 1-D amplitude spectra of Western and Eastern music are quite similar (Voss and Clarke, 1978), and that even stock price data, filtered with $1 /$ f noise and presented aurally, possess enough of the statistical regularity
} 
This study has bearing on the question of why art tends to reproduce the spatial statistical regularities of natural scenes. One view, which we call the affect hypothesis, suggests that natural, fractal-like statistics are inherently aesthetically pleasing and therefore artists should in general strive to match such statistics in their paintings (Redies, 2007; Redies et al., 2007; Spehar et al., 2003; Hagerhall et al., 2004). This is an intriguing proposal. Indeed, observers show relatively consistent preference over a range of 1-D fractal scaling exponents for artificial fractal images and for natural scene silhouettes (Aks and Sprott, 1996; Spehar et al., 2003; Sprott, 1993), though it should be noted that other studies have questioned the effect of fractal scaling on preference in urban design (Stamps, 2002).

Redies (2007), in particular, has articulated this hypothesis regarding universal aesthetics of natural statistics with appropriate nuance: "Fractal-like image properties may be necessary, but they are not sufficient to induce [a]esthetic perception." Redies (2007) has also argued that the bias towards natural scene-like statistics could extend to other affective responses, e.g., disgust or ugliness. That is, images with $1 /$ f structure may be judged to be more disgusting or ugly than random images (We are currently testing this notion in our lab). These affective responses are traditionally seen by philosophers as being components of aesthetic response, according to Redies.

Here we propose an alternative view, called the perceptibility hypothesis, which states that regularities in art statistics result ostensibly from the need to make an image that is readily visible to the human eye, and one that is amenable to creation by hand. The statistical regularities in art would thus be largely the result of the way pigment is typically applied to canvas, and of the way those marks are processed by the visual system.

Consider for example a comparison with white noise, which has a flat amplitude spectrum (slope of 0). Almost any collection of hand-made marks introduces spatial correlations, meaning that the resulting image would be likely to deviate from having a flat spectrum. A simple line will produce correlations; a patch of paint will do so to a greater extent. If a single line or paint stroke has the proper fractal structure, it alone can give rise to a $1 / f$ spectrum (see Field and characteristic of the Eastern hemisphere music to convince Chinese listeners that they are listening to music from Asia (Voss, 1985). 
Brady, 1997). We believe that art with spatial statistical regularities unlike those of natural scenes - e.g., white noise - is rare because of the challenges involved in producing such images by hand, not because such images are necessarily less aesthetically pleasing. The same logic holds for images with a spectrum much steeper than that of natural scenes (slope $>2$ ), as for example in a blurry image (see Fig. 4).

It is true that artists like Mondrian and Pollock have used application techniques that differ from traditional brush-based techniques and yet produced art whose statistical regularities match those typical of art as a whole. ${ }^{4}$ However, any technique that results in markings that resemble an array of locally correlated regions is likely to differ strongly from a technique that chooses tones randomly with respect to space (as in white noise). Despite the fact that random images comprise the great majority of possible images, we argue that artists are rarely able create images with such statistics using either their traditional or non-traditional paint application techniques. Therefore, our point is not that artists are forced to reproduce the spatial statistics of natural scenes, but that it is exceedingly hard work to avoid doing so without the assistance of a computer. Consider Attneave's classic paper on efficient sensory coding (1954), which testifies to the difficulty involved in creating a random image with a flat amplitude spectrum (i.e., white noise): Attneave employed two junior enlisted airmen to painstakingly fill in a grid of close to 20,000 small squares by hand based on values drawn from a random-number generator. We therefore suspect that few human artists have gone to the necessary trouble to escape the basic statistical regularities of scenes.

Even if one could easily produce images with "unnatural" spectra by hand, these images would not be perceptible in the way images with natural scene-like spectra are (see Knill et al., 1990), and such images would thus be rarely attempted. Indeed, there is evidence that the human visual system is well-adapted to the range of 2D fractal dimensions characteristic of natural

\footnotetext{
${ }^{4}$ Our tests on 3 digitized Pollock drip paintings from 1947-1950 [Scholar's Resource, Freeport, $\mathrm{ME}]$ found that their amplitude spectra had a mean slope of $-1.0 \pm 0.05$ (standard error); four Mondrian "compositions" [Scholar's Resource, Freeport, ME] had a mean slope of $-1.4 \pm 0.06$. These data were obtained with the same procedure used elsewhere in the paper. The Mondrian images were nearly square, while the Pollock images were cropped to make them square for testing.
} 
scenes, which closely matches that for art (Knill, et al., 1990; Field and Brady, 1997; Parraga et al., 2000; Billock, 2000; Graham et al., 2006).

[Fig. 4]

Moreover, regularities in amplitude spectrum slope hardly constrain the space of possible images that the affect hypothesis would judge to be aesthetically pleasing, nor does this hypothesis account for artistic styles that do not match the amplitude spectra of natural scenes (e.g., monochromes). In addition, nonlinear luminance compression in paintings fundamentally alters intensity statistics for art compared to the natural world and therefore art is unlikely to "induce a specific resonant state in the visual system...based on the adaptation of the visual system to natural scenes." (Redies, 2007). Nonlinear luminance compression strategies reflect the need to make an image that is perceptible to the eye as depicting the world. We are currently exploring statistical regularities in these compression strategies across artists.

To our knowledge, no studies have addressed whether there is a similar range of preference for 2-D fractal dimensions in images. We use the term '2-D fractal dimension' in reference to images whose greyscale intensities vary continuously across two spatial dimensions. The term '1-D fractal dimension' pertains to images comprised entirely of binary boundaries. In other words, the 1-D fractal dimension measures the degree to which a 1-D boundary fills up 2-D space, while the 2-D fractal dimension measures the degree to which a 2-D intensity surface fills up 3-D space. All fractal images tested in Spehar et al. (2003) are 1-D by this definition, though it should be noted that the 'control' images presented in Fig. 7 in that paper were 2-D by this definition. These control images — which we presume are derived from white noise - showed no statistical difference in preference.

If preference were found to be limited to a range of 2-D fractal dimensions, this would not by itself invalidate the perceptibility hypothesis, since this hypothesis proposes that art is rarely statistically random because random images are difficult to perceive and to produce. That is, we do not predict a preference for one 2-D fractal dimension over another, except in the sense that the preference will be lower for images lacking 1/f scaling. As we have noted, random images do 
not show 1/f scaling, yet they comprise the great majority of possible images. We believe that visual system coding is matched to natural spatial statistics such that viewers find random images to be equally indiscriminable, while images with $1 /$ f scaling stand a better chance of being perceived and preferred. We remain agnostic as to the amount of aesthetic pleasure that is to be derived from images possessing a particular fractal dimension - the present study concerns only the high prevalence of art with 2-D amplitude spectra that are well described by $1 / \mathrm{f}$, not such images' relative aesthetic quality.

As noted in Graham and Field (2007b), artists have occasionally strayed from the statistical regularities of natural scene amplitude spectra - as well as the limitation on the dynamic range of luminances - and yet produced fine art. Statistical differences in spectra and in pixel intensity distributions for representational and non-representational art, and for Eastern and Western art, are also subject to exceptions.

Finally, we note that regularities in the amplitude spectra are only one of many types of redundancy in the spatial statistics of images. Chandler and Field (2007) have estimated that around $40 \%$ of the statistical predictability of natural images is due to regularities in the amplitude spectra. It is unclear at present the extent to which higher order redundancies of natural scenes are reproduced in paintings, nor is it known how these statistics vary across painting type and provenance.

\section{CONCLUSION}

It seems sensible that paintings - which are created in order to be viewed by the human visual system — reproduce the statistical regularities of the world (Graham and Field, 2007b, Redies et al., 2007). Moreover, as Graham and Field showed, paintings as a group show spatial statistics which are quite similar to those of natural scenes despite the need for painters to impose massive nonlinear compression with respect to luminance. Here we have shown that various types of human art work - including abstract works — show essentially the same basic amplitude spectrum shape. However, different classes of art also vary systematically across a number of statistical measures that are relevant to the efficient coding of statistically regular natural scenes. In particular, works from the Eastern hemisphere showed significantly more negatively skewed 
intensity distributions than works from the Western hemisphere, a difference attributed to systematic differences in background tone. Eastern works also showed a higher proportion of fine detail compared to Western works in our sample. In addition, abstract works showed a higher proportion of fine detail compared to representational works in our sample, though abstract works followed the general regularities of representational works and natural scenes. Similarities between basic statistics for art of all kinds and for natural scenes are viewed by the perceptibility hypothesis as a necessary byproduct of the need to create an image that is readily perceived by the eye and of the nature of manual pigment application techniques.

\section{REFERENCES}

Aks, D. and Sprott, J. C. (1996). Quantifying aesthetic preference for chaotic patterns. Empirical Studies of the Arts 14, 1-16.

Atick, J. J. and Redlich A. N. (1992). What does the retina know about natural scenes? Neural Comput. 4, 196-210.

Attneave, F. (1954). Some informational aspects of visual perception. Psychol. Rev. 61, 183-193.

Balboa, R. M., Tyler, C. W. and Grzywacz, N. M. (2001). Occlusions contribute to scaling in natural images. Vision Res. 41, 955-964.

Bell, A. J. and Sejnowski, T. J. (1997). The 'independent components' of natural scenes are edge filters. Vision Res. 37, 3327-3338.

Billock, V. A. (2001). Neural acclimation to $1 / \mathrm{f}$ spatial frequency spectra in natural images transduced by the human visual system. Physica D 137, 379-391.

Burton, G.J. and Moorhead, I. R. (1987). Color and spatial structure in natural scenes. Appl. Optics 26, 157-170.

Chandler, D. M. and Field, D. J. (2007). Estimates of the information content and dimensionality of natural scenes from proximity distributions. J. Opt. Soc. Am. A 24, 922-941.

Field, D. J. (1987). Relations between the statistics of natural images and the response profiles of cortical cells. J. Opt. Soc. A. 4, 2379-2394.

Field D. J. (1993). Scale-invariance and Self-similar 'Wavelet' Transforms: an Analysis of Natural Scenes and Mammalian Visual Systems. In: Wavelets, Fractals and Fourier Transforms: New Developments and New Applications. Oxford: Oxford University Press.

Field, D. (1994). What is the goal of sensory coding? Neural Comput. 6, 559-601.

Field, D. J. and Brady, N. (1997). Visual Sensitivity, Blur and the Sources of Variability in the Amplitude Spectra of Natural Scenes. Vision Res. 37, 3367-3383.

Frazor, R. A. and Geisler, W. A. (2006). Local luminance and contrast in natural images. Vision Res. 46, 1585-1598.

Graham, D. J., Chandler, D. M. and Field, D. J. (2006). Can the theory of "whitening'" explain the center-surround properties of retinal ganglion cell receptive fields? Vision Res. 46, 29012913. 
Graham, D. J. and Field, D. J. (2007a). Efficient neural coding of natural images. In New Encyclopedia of Neuroscience, L. R. Squire, ed. London: Elsevier (in press).

Graham, D. J. and Field, D. J. (2007b). Statistical regularities of art images and natural scenes: spectra, sparseness and nonlinearities. Spatial Vision (in press).

Hagerhall, C. M., Purcell, T. and Taylor, R. (2004). Fractal dimension of landscape silhouette outlines as a predictor of landscape preference. J. Enivonmental Psych. 24, 247-255.

Knill, D. C., Field, D. and Kersten, D. (1990). Human discrimination of fractal images. J. Opt. Soc. Am. A 7, 1113-1123.

Laughlin, S. B. (1981). A simple coding procedure enhances a neuron's information capacity. $Z$. Naturforsch. 36C, 910-912.

Motoyoshi, I., Nishidam S., Sharan, L. and Adelson, E. H. (2007). Image statistics and the perception of surface qualities. Nature 447, 206-209.

Olshausen, B. A. and Field, D. J. (1996). Emergence of simple-cell receptive field properties by learning a sparse code for natural images. Nature 381, 607-609.

Parraga, C. A., Troscianko, T. and Tolhurst, D. J. (2000). The human visual system is optimised for processing the spatial information in natural visual images. Curr. Biol. 10, 35-38.

Redies, C. (2007). A universal model of esthetic perception based on the sparse and efficient coding of natural stimuli. Spatial Vision (in press).

Redies, C., Hasenstein, J., and Denzler, J. (2007). Fractal-like image statistics in visual art: similarity to natural scenes. Spatial Vision (in press).

Rogowitz, B. E. and Voss, R. F. (1990). Shape perception and low-dimensional fractal boundaries. Proc. SPIE 1249, 387-394.

Ruderman, D. L. (1997). Origins of scaling in natural images. Vision Res. 37, 3385-3398.

Spehar, B., Clifford, C. W. G., Newell, B. R. and Taylor, R. P. (2003). Universal aesthetics of fractals. Computers and Graphics 27, 813-820

Sprott, J. C. (1993). Automatic generation of strange attractors. Computer \& Graphics 17, 325-32.

Srinivasan, M. V., Laughlin, S. B. and Dubs, A. (1982). Predictive coding: a fresh view of inhibition in the retina. Proc. R. Soc. B Biol. Sci. 216, 427-459.

Stamps, A. E. (2002). Fractals, skylines, nature and beauty. Landscape and Urban Planning 60, 163-184.

Taylor, R. P., Micolich, A. P. and Jonas, D. (1999). Fractal Expressionism. Physics World 12, 25-29.

Tolhurst, D. J., Tadmor, Y. and Chao, T. (1992). The amplitude spectra of natural images. Ophthalmic Physiol. Opt. 12, 229-232.

Torralba, A. and Oliva, A. (2003). Statistics of natural image categories. Netw.: Comput. Neural Syst. 14, 391-412.

Voss, R. F. (1985). Random fractal forgeries. In Fundamental Algorithms for Computer Graphics, R. A. Earnshaw, ed. New York: Springer-Verlag.

Voss, R. F. and Clarke, J. (1978). 1/f noise in music: Music from 1/f noise. J. Acoust. Soc. Am. 63, 258-263.

\section{ACKNOWLEDGEMENTS:}


DJG was supported by NEI grant EY015393 and DJF was supported by NGA Contract HM 1582-05-C-0007. We wish to acknowledge the many current and former staff of the Herbert F. Johnson Museum of Art in Ithaca, NY, who made this work possible: Frank Robinson, (director), Julie Magura (chief photographer), Whitney Tassie, Elizabeth Emrich (rights and reproductions), Renee Grinnell (rights and reproductions), Oya Rieger (Olin Library, Cornell), Rhea Garen (Olin Library, Cornell), Mike Bevans (Academic Imaging, Inc.), and Peter Hirtle.

\section{FIGURES}

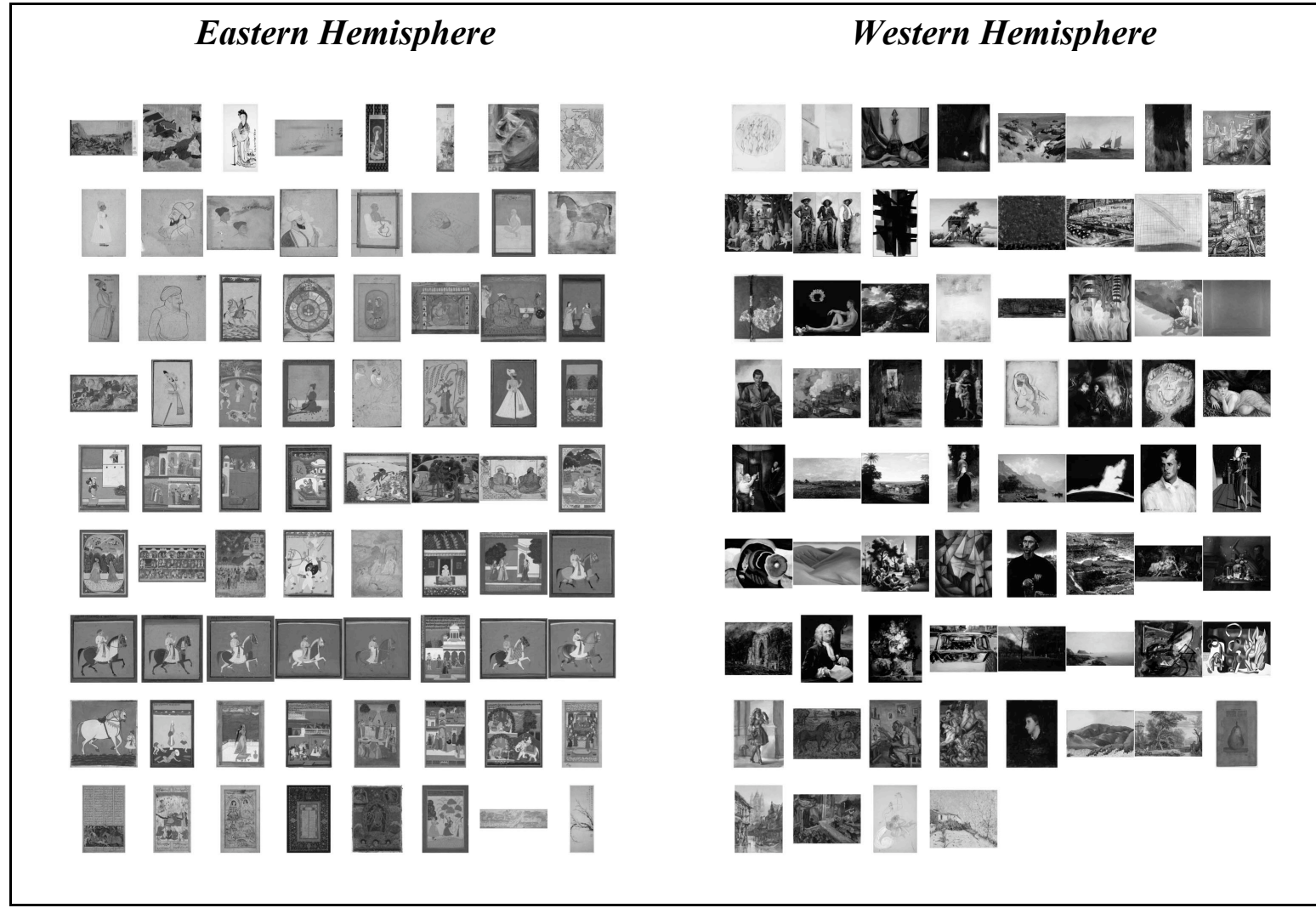

FIGURE 1. Images as classified according to provenance (place of origin). All images are courtesy of the Herbert F. Johnson Museum of Art, Cornell University.

Landscape Portrait/Still-life Abstract




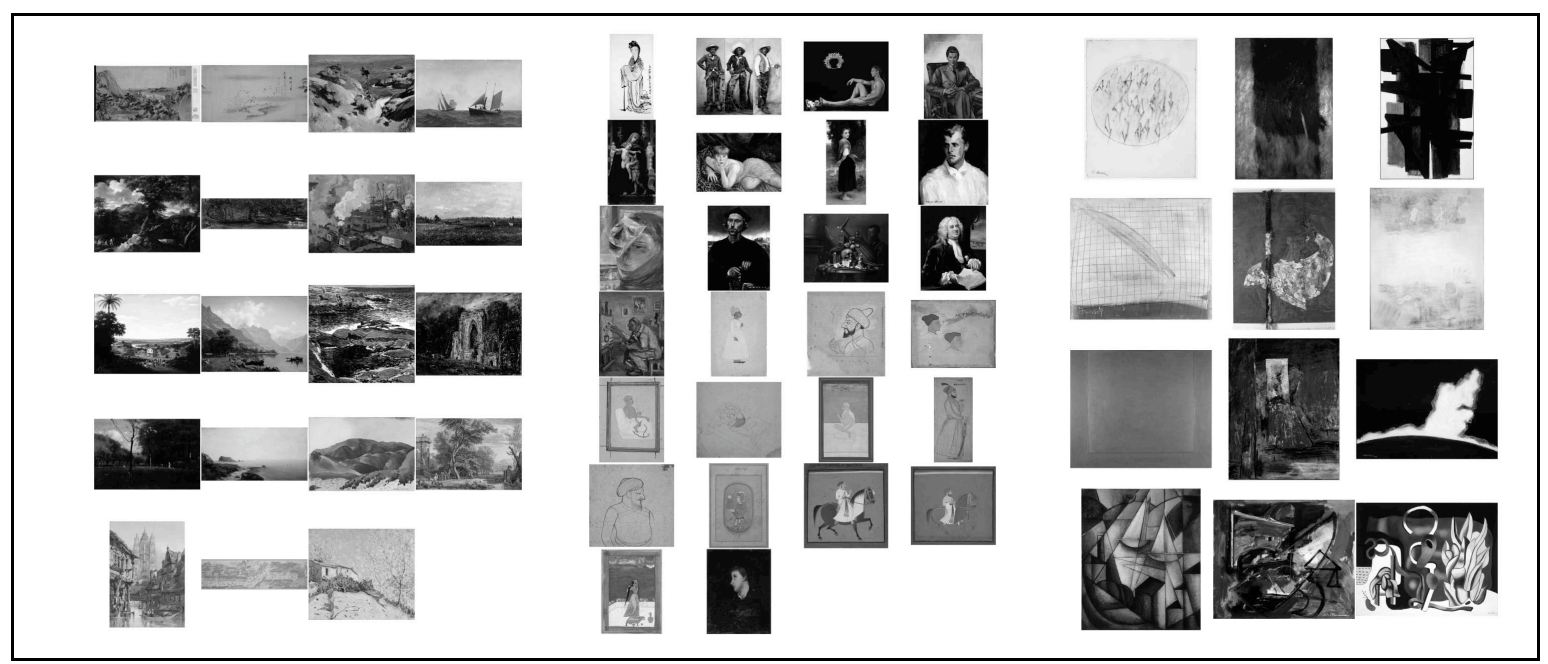

FIGURE 2. Image categories (from right to left: landscape, portrait/still-life, abstract) as determined by the agreement of 6 judges using a forced-choice paradigm. All images are courtesy of the Herbert F. Johnson Museum of Art, Cornell University.

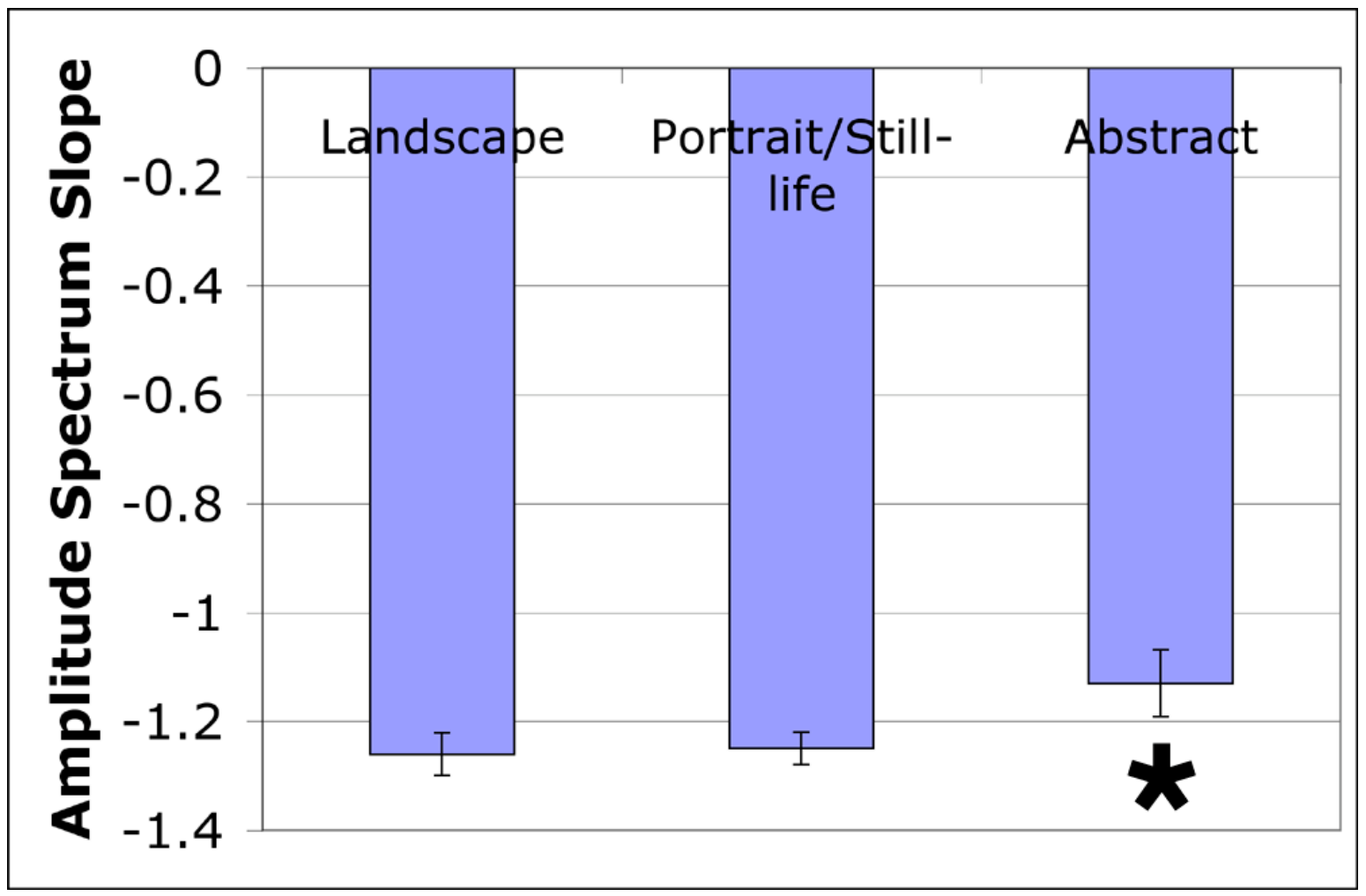

FIGURE 3. Plot of mean amplitude spectrum slope by image category. Abstract works were significantly different from the other two classes taken together $(\mathrm{p}<0.03)$. Abstract works also approached significance with both the Landscape and the Portrait/Still-life categories individually, with $\mathrm{p}=0.058$ and $\mathrm{p}=0.054$, respectively. Error bars show standard error. 

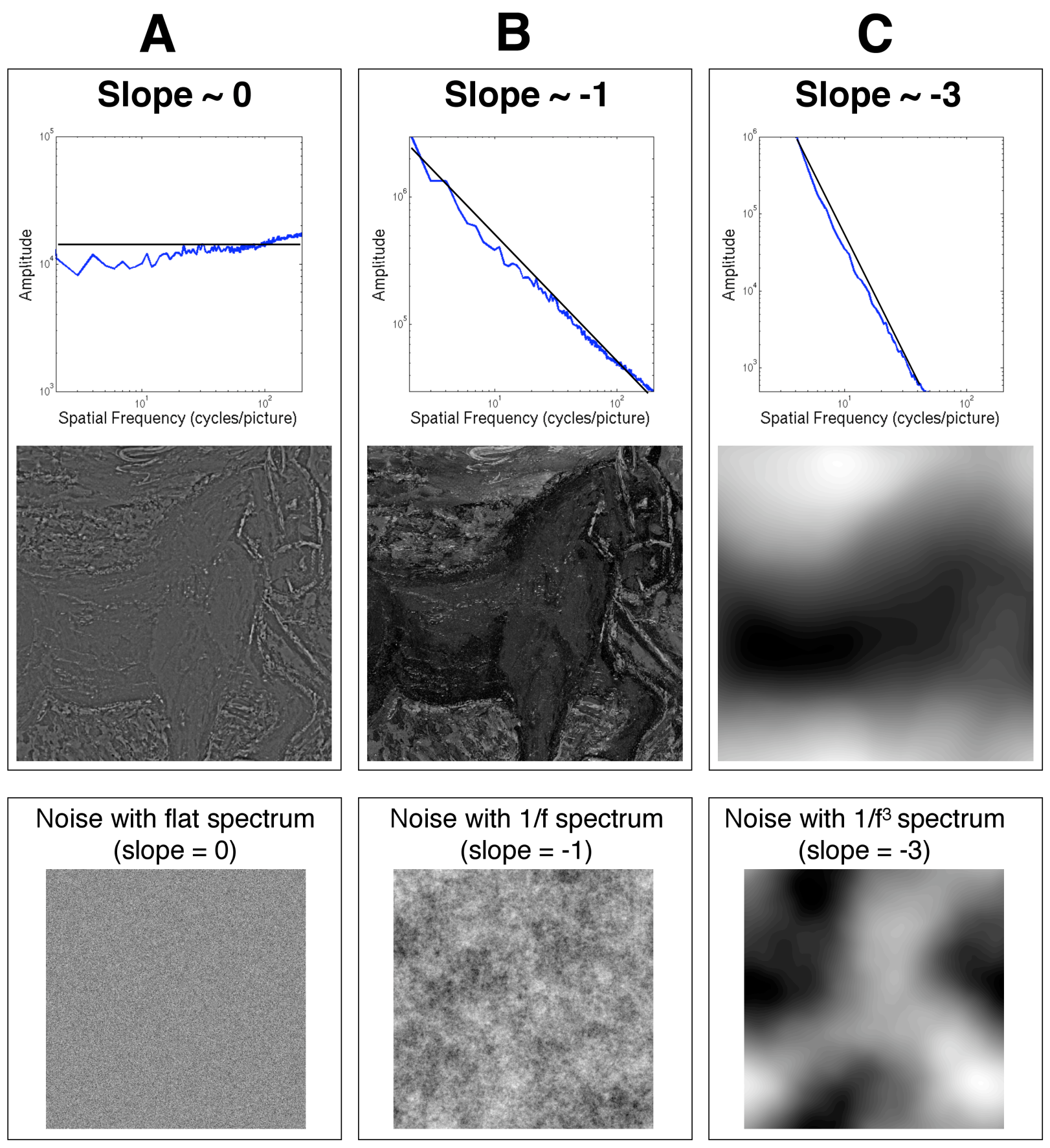

FIGURE 4. Examples of images with spatial frequency amplitude spectrum slope of approximately 0 (A), -1 (B) and 3 (C). The amplitude spectrum for a portion the painting Untitled (Woman with Red Horse) is plotted on log-log axes. Below the spectrum is the portion of the image for which the spectrum was calculated. (A) and (C) were generated by adjusting the spectrum of the original image (B) in the frequency domain. Gaussian-distributed noise with amplitude spectra similar to those of the corresponding paintings are also shown. Painting is courtesy of the Herbert F. Johnson Museum of Art, Cornell University: David Burliuk, Untitled (Woman with Red Horse), 1951, Gift of Hans Namuth accession. 